\title{
Biographie du $D^{r}$ Ferrière.
}

de finesse, de tact et de possession de soi, rendit à la vie active et utile bien des personnalités qui, sans lui, eussent été enfermées à jamais dans des asiles. Il fut aussi, par excellence, le médecin de famille, celui qui écoute toutes les doléances et trouve une issue à tous les conflits non seulement des corps, mais des âmes. Jamais on ne saura le nombre de ces êtres repêchés par lui, remis sur la voie droite, qui lui vouent une reconnaissance éternelle et qui pleurent aujourd'hui son départ. "

Et qu'il nous soit permis de terminer cette esquisse biographique incomplète par ce portrait physique que Noëlle Roger a tracé dans l'article qu'elle a consacré au $D^{r}$ Ferrière dans la Semaine littéraire :

"Mince, pâle et fin, il avait un beau visage d'ascète, encadré d'une barbe qui était devenue toute blanche, un grand front calme sous les cheveux argentés, des yeux clairs, un regard aigu et doux, si brillant d'esprit, parfois, et si profondément pénétrant qu'on avait le sentiment que les paroles étaient inutiles. Ce regard savait saisir en vous la pensée, l'inquiétude, la souffrance, et il se voilait aussitôt de sympathie délicate qu'il n'était pas besoin d'exprimer non plus... »

\section{Frédéric Ferrière médecin.}

F. A. Ferrière après avoir fait ses études à Genève, fréquenta pour y apprendre la médecine, les universités de Berne, Heidelberg et Vienne. Dans l'antique Alma mater du Grand Duché de Bade, il fut l'élève de maîtres célèbres, tels que Arnold, Gegenbauer, Simon et Friedreich; à Vienne, de Billroth, d'Hébra, etc. Ce fut à Heidelberg qu'il acquit le grade de docteur en médecine, à la suite de la présentation de sa thèse sur : "Des procédés opératoires contre le prolapsus utérin". Ce travail, qui date de 1875, est une revue complète de tout ce qui s'était fait jusqu'alors dans ce domaine ; travail d'une documentation sûre, d'une saine logique et d'une critique qui dénote chez ce jeune médecin un esprit mûr, prêt aux déductions raisonnées, 


\section{Biographie du Dr Ferrière.}

donnant déjà l'impression de ce qu'il serait plus tard. En effet, tout ce que nous connaissons de sa vie médicale est le reflet d'une âme aux idées élevées, mettant les dons de l'intelligence au-dessus de tout; noble nature, secondée dans l'exercice de son art par une faculté d'intuition remarquable. C'est ce qui lui permit d'être avant tout un médecin de famille, au sens le plus élevé du mot. Il le resta durant toute son activité professionnelle. Ses études l'avaient d'ailleurs poussé à consacrer à la gynécologie un intérêt particulier et, grâce à une grande expérience, jointe à beaucoup de tact, il jouit, comme accoucheur, d'une réputation méritée.

Pendant la guerre de I870, en sa qualité d'engagé volontaire dans une ambulance, il fit sa première éducation pratique de médecin militaire. A peine âgé de 22 ans, il dirigea seul de nombreux transports de blessés à travers la Haute-Saône et les Vosges. Il y acquit sa maîtrise dans l'art d'organiser les évacuations.

De 1875 à I 876 , à l'occasion de son expédition au Monténégro, le $\mathrm{D}^{\mathrm{r}}$ Ferrière, ayant terminé sa mission diplomatique auprès du prince Nicolas, se rendit, accompagné de l'un de ses collègues, à la demande du prince, dans le nord du pays, à la frontière turque, à Sveti-Luca, près de Nikchitsch, pour y diriger un petit hôpital qui recevait les blessés de combats incessants. Il y soigna pendant trois mois les victimes de l'insurrection herzégovienne. Il eut égalcment à secourir les 60 à 70,000 réfugiés herzégoviens qui avaient fui au Monténégro et y doublaient presque le chiffre de la population à cette époque. F. A. Ferrière put, là, metîre à profit son expérience, et le grand nombre des blessés et malades qu'il recueillit témoigne de la belle qualité de ses méthodes d'improvisation sur les champs de bataille et d'organisation des secours.

On a pu lire au cours de sa vie de nombreux articles publiés par le Bulletin international de la Croix-Rouge, se rapportant à ia médecine, à la chirurgie, à l'hygiène militaire ${ }^{1}$.

1 Voy. la bibliographie ci-dessous. 


\section{Biographie du Dr Ferrière.}

Rentré à Genève, il se fait remarquer par son initiative à combattre les défauts de l'hygiène publique.

En I885, il fait un rapport publié par la société d'Hygiène de Genève sur "les visites sanitaires des immeubles", travail tout de conscience et de franchise, qui révèle les imperfections considérables de la ville de Genève à cette époque, et le chemin parcouru depuis lors, grâce au mouvement que cette compilation savante sut déclencher. En 1887 , il présente à Vienne, au $6^{\mathrm{me}}$ congrès international pour l'hygiène et de la démographie, un travail en qualité de secrétaire de la société d'Hygiène de Genève. Le "Rapport concernant la Suisse, en réponse au programme remis, lors du congrès de La Haye, à chacun des membres de la Commission chargée de l'enquête sur les falsifications des denrées alimentaires " est un exposé complet de la surveillance et des moyens chimiques d'examen dont on dispose pour se garantir contre ces fraudes. Mais il y aurait de grandes difficultés à vouloir réglementer par des accords internationaux des mesures d'hygiène nationale. Quel serait le droit international qui sanctionnerait les mesures édictées?

En 1897, il fait une conférence à la société des samaritains de Genève sur "Les maisons insalubres". Aussitôt la société pour l'Amélioration du logement, qu'il présida à plusieurs reprises, publie cette plaquette, où le $D^{r}$ Ferrière passe en revue tous les défauts de la construction en général, énumère les dangers des logements malsains et étale au grand jour les causes de négligences et d'imperfections, cherchant à engager ainsi nos autorités à en préserver la population pour l'avenir.

En I899, il insiste comme député au Grand Conseil de Genève sur "L'hygiène intellectuelle dans l'instruction secondaire". Dans cette brochure, dont un exemplaire a été envoyé à chacun des régents secondaires de la Suisse, sur la demande du bureau sanitaire fédéral, l'auteur fait un exposé des modalités rationnelles permettant une utilisation meilleure des forces physiques avec un rendement psychique supérieur. Il y révèle les lacunes non seulement matérielles, mais surtout psychologiques de nos écoles. Reprenant les statistiques récentes du 


\section{Biographie du Dr Ferrière.}

physiologiste suédois Axel Key (oncle de l'écrivain bien connu Ellen Key, qui fut et reste une de ses admiratrices les plus ferventes), il esquisse le tableau de ce que doit être l'école, si elle tient, comme c'est son devoir, à sauvegarder chez l'enfant une âme saine dans un corps en bonne santé. Par là il se montre le précurseur de ces écoles nouvelles à la campagne dont il soutint, dès cette époque, les premiers pas et auxquelles il devait porter plus tard un intérêt très actif.

En Igor, dans un travail lu à l'Institut national genevois, le Dr Ferrière expose "La lutte contre la tuberculose à Genève". Cette mise au point complète de la question, recommandant le traitement de la maladie par la prophylaxie et l'hygiène, avait pour but de soutenir les idées du moment à Genève, notamment la création d'un sanatorium populaire, et rappelait au grand public son devoir contre les dangers de la tuberculose. On comprend aisément pourquoi le Comité international de la Croix-Rouge désigna le Dr Ferrière pour présenter à la VIIIme Conférence internationale de la Croix-Rouge, à Londres I907, le "Rapport sur la participation de la Croix-Rouge à la lutte contre la tuberculose ».

Ce mémoire valut au rapporteur la croix de la Légion d'honneur.

Bien que miné dans sa santé physique, le $\mathrm{D}^{\mathrm{r}}$ Ferrière conserva intactes jusqu'au dernier moment ses qualités intellectuelles. Dans son dernier travail, la plaquette "Hommage à Pasteur", il retraça la vie du savant avec une élévation de pensée et une tenue morale dignes de celui auquel elles s'adressaient. Et luimême jusqu'à la fin de sa vie justifia cette parole de Pasteur : "Il me semble que je commettrais un vol si je passais une journée sans travailler".

Dr A. R.

\section{Le $D^{r}$ Fréderic Ferrière et la Croix-Rouge.}

Lorsque mourut, en aoút I9 1o, Gustave Moynier, après 40 ans de présidence du Comité international de la Croix-Rouge, on put à juste titre dire de lui que, tracer sa biographie, c'était 